\title{
THE INVERSE CONDUCTIVITY PROBLEM WITH ONE MEASUREMENT: UNIQUENESS FOR CONVEX POLYHEDRA
}

\author{
BARTOLOMÉ BARCELÓ, EUGENE FABES, AND JIN KEUN SEO
}

(Communicated by Barbara L. Keyfitz)

\begin{abstract}
Let $\Omega$ denote a smooth domain in $R^{n}$ containing the closure of a convex polyhedron $D$. Set $\chi_{D}$ equal to the characteristic function of $D$. We find a flux $g$ so that if $u$ is the nonconstant solution of $\operatorname{div}\left(\left(1+\chi_{D}\right) \nabla u\right)=0$ in $\Omega$ with $\frac{\partial u}{\partial n}=g$ on $\partial \Omega$, then $D$ is uniquely determined by the Cauchy data $g$ and $f \equiv u / \partial \Omega$.
\end{abstract}

\section{INTRODUCTION}

Let $\Omega$ be a bounded domain in $R^{n}, n \geq 2$, with a connected boundary and $D$ a subdomain in $\Omega$. Assume both $\Omega$ and $D$ are conductors of electricity. We consider the following question: Can we set up a magnetic field $\vec{E}$ surrounding $\Omega$ with a known flux $g$ across $\partial \Omega$ so that calculating the potential of the field on $\partial \boldsymbol{\Omega}$ will determine $D$ ?

Writing $\vec{E}=\nabla u$ we have

$$
\begin{aligned}
& L_{\gamma} u=\operatorname{div}(\gamma(x) \nabla u)=0 \text { in } \Omega, \\
& \frac{\partial u}{\partial n}=g \quad \text { on } \partial \Omega,
\end{aligned}
$$

where $\gamma(x)$ is the conductivity and $\frac{\partial u}{\partial n}$ denotes the normal derivative of $u$ on $\partial \Omega$. The question now becomes: Can we choose $g$ so that $g$ and $f=\left.u\right|_{\partial \Omega}$ uniquely determine $D$ ?

For the sake of definiteness we take $\gamma(x)=1+\chi_{D}(x)$, where $\chi_{D}$ denotes the characteristic function of $D$. In this case, Friedman and Isakov [1] proved that there is a flux $g$ so that $g$ and $f$ uniquely determine $D$ if $D$ is assumed to be a convex polyhedron situated away from the boundary of $\Omega$, that is, if

$$
\operatorname{diam}(D)<\operatorname{dist}(D, \partial \Omega) .
$$

In this paper we are able to remove this extra condition. Specifically, we prove the following uniqueness result: There exists a function $g$ defined on

Received by the editors August 19, 1992 and, in revised form, December 10, 1992; contents presented at an international meeting titled Partial Differential Equations of Elliptic Type held in Cortona, Italy, October 12-16, 1992, and sponsored by Istituto Nazionale di Alta Matematica and Consiglio Nazionale delle Ricerche.

1991 Mathematics Subject Classification. Primary 35R30, 35R05, 35B30. 
$\partial \Omega$ such that if $D_{1}, D_{2}$ are convex polyhedra compactly contained in $\Omega$ and $u_{i}, i=1,2$, is the solution of

$$
\begin{gathered}
\operatorname{div}\left(\left(1+\chi_{D_{i}}(x)\right) \nabla u_{i}(x)\right)=0 \text { in } \Omega, \\
\int_{\Omega} u_{i} d x=0 \text { and }\left.\frac{\partial u_{i}}{\partial n}\right|_{\partial \Omega}=g,
\end{gathered}
$$

then if $\left.u_{1}\right|_{\partial \Omega}=\left.u_{2}\right|_{\partial \Omega}$, we conclude $D_{1}=D_{2}$. We prove this result in $\S 3$ (Theorem 1) for the case of convex polygons. The proof for convex polyhedra in $R^{n}$ follows from Lemma 3 using similar arguments.

\section{Preliminaries}

We recall that by definition a convex polyhedron $\mathscr{P}$ in $R^{n}$ is a finite intersection of half spaces. That is, if

$$
H_{i}=\left\{x \in R^{n}:\left(x-x_{i}\right) \cdot n_{i}>0\right\}
$$

for some points $x_{1}, x_{2}, \ldots, x_{k} \in R^{n}$ and unit vectors $n_{1}, n_{2}, \ldots, n_{k} \in R^{n}$, then

$$
\mathscr{P}=H_{1} \cap H_{2} \cap \cdots \cap H_{k} .
$$

An edge $\gamma$ of the polyhedron $\mathscr{P}$ is the intersection of two faces, i.e., the intersection of two hyperplanes used in the definitions of $\mathscr{P}$. A polyhedron $D$ in $R^{n}$ is an open connected set that is the union of convex polyhedrons.

Given a bounded $C^{2}$ domain $\Omega$ in $R^{n}$ and a polyhedron $D$ compactly supported in $\Omega$, let us consider the Neumann problem

$$
\left\{\begin{array}{l}
L u=\operatorname{div}\left(\left(1+\chi_{D}(x)\right) \nabla u\right)=0 \quad \text { in } \Omega, \\
\frac{\partial u}{\partial n}=g \quad \text { on } \partial \Omega,
\end{array}\right.
$$

where $n$ is the unit normal to the boundary and $u$ is normalized by $\int_{\Omega} u=0$.

By a weak solution $u$ to $L u=0$ we mean a function $u \in L^{2}(\Omega)$ whose partial derivatives $u_{x_{i}}$, in the distributional sense, belong to $L^{2}(\Omega)$ and such that

$$
\sum_{i=1}^{n} \int_{\Omega}\left(1+\chi_{D}\right) \frac{\partial u}{\partial x_{i}} \frac{\partial \phi}{\partial x_{i}} d x=0
$$

for all $\phi \in C_{0}^{\infty}(\Omega)$, the class of $C^{\infty}$ functions with compact support in $\Omega$.

It is well known that if $g \in L^{\infty}(\partial \Omega)$, then there exists a unique solution $u$ to (1) such that $u \in C^{\alpha}(\bar{\Omega})$ for some $\alpha>0$.

If we set

$$
u^{e}=\left.u\right|_{\Omega \backslash D} \text { and } u^{i}=\left.u\right|_{D},
$$

then $\Delta u^{e}=0$ in $\Omega \backslash \bar{D}, \Delta u^{i}=0$ in $D$, and $u^{e}=u^{i}$ on $\partial D$.

Also, by integrating by parts in (1) we get

$$
\frac{\partial u^{e}}{\partial n}=2 \frac{\partial u^{i}}{\partial n}
$$

on the smooth part of $\partial D$.

\section{THE MAIN THEOREM}

Let $\Omega \subset R^{n}$ be a bounded convex $C^{2}$ domain in $R^{n}$ with a connected boundary. Let $D_{1}, D_{2}$ be two polyhedra compactly supported in $\Omega$. For 
$i=1,2$ consider

$$
\left\{\begin{array}{l}
L_{\gamma_{i}} u_{i}=\operatorname{div}\left(\left(1+\chi_{D_{i}}(x)\right) \nabla u_{i}\right)=0 \quad \text { in } \Omega, \\
\frac{\partial u_{i}}{\partial n}=g \quad \text { on } \partial \Omega,
\end{array}\right.
$$

where we also take $\int_{\Omega} u_{i} d x=0$.

Let us fix a $L^{\infty}(\partial \Omega)$ function $g$ such that there is no harmonic function $u$ on any neighborhood $\mathscr{V}$ of any point of $\partial \Omega$ with $g=\nabla u \cdot n$ on $\mathscr{V} \cap \partial \Omega$. For example, let us take a dense sequence of points $\left\{p_{k}\right\}$ on the surface boundary $\partial \Omega$ and consider the function

$$
g(x)=\sum_{k=1} 2^{-k}\left|x-p_{k}\right|-c,
$$

where $x \in R^{n}$ and $c$ is chosen so that $\int_{\partial D} g d \sigma=0$. The function $g$ is Lipschitz but not $C^{1}$ on any surface ball $B(p) \cap \partial \Omega$. If $u$ is harmonic in $B(p)$, then $\nabla u \cdot n$ is $C^{1}(B(p)) \cap \partial \Omega$, so we cannot have $\nabla u \cdot n=g$ there.

Theorem 1. Let $D_{1}, D_{2}$ be convex polygons in $R^{2}$. Assume $u_{i}, i=1,2$, are the solutions to the Neumann problem (4) with $g$ as in (5). If $u_{1}=u_{2}$ on $\partial \Omega$, then $D_{1}=D_{2}$.

Proof. If $D_{1}$ and $D_{2}$ have the same corners, there is nothing to be proved. Let us assume that $P$ is a corner of $D_{1}$ but $P$ is not a corner of $D_{2}$.

We can also assume, for simplicity, that $P$ is the origin of $R^{2}$ and, in polar coordinates, if $B_{r_{0}}$ is a small ball centered at the origin,

$$
B_{r_{0}} \cap D_{1}=\left\{(r, \theta): 0<r<r_{0}, 0<\theta<\theta_{0}\right\}
$$

and

$$
B_{r_{0}} \cap D_{2}=\varnothing \text {. }
$$

Note that $0<\theta_{0}<\pi$ because of the convexity of $D_{1}$.

Since $u_{1}=u_{2}$ on $\partial \Omega$ and also $\frac{\partial u_{1}}{\partial n}=\frac{\partial u_{2}}{\partial n}=g$ on $\partial \Omega$, by the unique continuation property for harmonic functions it follows that $u_{1}=u_{2}$ on the connected component of $\Omega \backslash\left(D_{1} \cup D_{2}\right)$ near the boundary $\partial \Omega$. Since $u_{2}$ is harmonic on $B_{r_{0}},\left.u_{1}\right|_{B_{r_{0}} \backslash D_{1}}$ has a harmonic extension to the whole ball $B_{r_{0}}$. (In fact, this harmonic extension is $u_{2}$ itself.) Let us call $u_{1}^{\text {ext }}$ this harmonic extension.

From Lemma 3 below, in polar coordinates, we have

$$
u_{1}^{\mathrm{ext}}(r, \theta)=u_{1}^{\mathrm{ext}}\left(r, \theta \pm 2 \theta_{0}\right)
$$

for all $(r, \theta) \in B_{\epsilon}$, for some $0<\epsilon<r_{0}$.

Let us represent by $\tilde{u}_{1}$ the rotation of $u_{1}$ by the angle $2 \theta_{0}$, that is,

$$
\tilde{u}_{1}(r, \theta) \equiv u_{1}\left(r, \theta-2 \theta_{0}\right) \text {, }
$$

and let us denote by $\tilde{\Omega}, \tilde{D}_{1}$ the rotated corresponding domains; that is,

$$
\begin{aligned}
& \tilde{\Omega}=\left\{\left(r, \theta+2 \theta_{0}\right):(r, \theta) \in \Omega\right\}, \\
& \tilde{D}_{1}=\left\{\left(r, \theta+2 \theta_{0}\right):(r, \theta) \in D_{1}\right\} .
\end{aligned}
$$

Since

$$
\begin{aligned}
& u_{1}(r, \theta)=u_{1}^{\text {ext }}(r, \theta) \quad \text { for }(r, \theta) \in B_{\epsilon} \backslash D_{1}, \\
& \tilde{u}_{1}(r, \theta)=u_{1}^{\text {ext }}\left(r, \theta-2 \theta_{0}\right) \quad \text { for }(r, \theta) \in B_{\epsilon} \backslash \tilde{D}_{1},
\end{aligned}
$$


it follows from (6) that

$$
u_{1}=\tilde{u}_{1} \quad \text { in }\left(B_{\epsilon} \backslash D_{1}\right) \cap\left(B_{\epsilon} \backslash \tilde{D}_{1}\right) .
$$

Now, by unique continuation, we have $u_{1}=\tilde{u}_{1}$ on all open connected components near the origin of $(\Omega \cap \tilde{\Omega}) \backslash\left(D_{1} \cup \tilde{D}_{1}\right)$.

Since $\theta_{0}<\pi$, there are $\theta_{1}$ and $\delta>0$ such that if $\theta_{1}<\phi<\theta_{1}+\delta$, then the ray

$$
T_{\phi}=\{(r, \theta): r>0, \theta=\phi\}
$$

does not intersect $D_{1} \cup \tilde{D}_{1}$. Let $Q_{\phi}$ and $\tilde{Q}_{\phi}$ be the points on $\partial \Omega$ and $\partial \Omega$ respectively such that

$$
\left(0, Q_{\phi}\right) \subset \Omega \cap T_{\phi} \quad \text { and } \quad\left(0, \tilde{Q}_{\phi}\right) \subset \tilde{\Omega} \cap T_{\phi},
$$

where $\left(0, Q_{\phi}\right)$ denotes the open line segment joining the origin 0 with $Q_{\phi}$ and $\left(0, \tilde{Q}_{\phi}\right)$ the open line segment joining 0 with $\tilde{Q}_{\phi}$. We will prove

$$
Q_{\phi}=\tilde{Q}_{\phi} \quad \text { for all } \theta_{1}<\phi<\theta_{1}+\delta \text {. }
$$

If not, we may assume $\left(0, Q_{\phi}\right) \subset\left(0, \tilde{Q}_{\phi}\right)$. Since $u_{1}$ and $\tilde{u}_{1}$ coincide on $\left(0, Q_{\phi}\right)$ and $\tilde{u}_{1}$ is harmonic in a neighborhood of $Q_{\phi}$, a contradiction follows because $u_{1}$ cannot be harmonically extended across any small neighborhood of the point $Q_{\phi}$ because of the bad boundary data $g$. This proves that if

$$
\mathscr{S}=\bigcup_{\theta_{1}<\phi<\theta_{1}+\delta}\left(0, Q_{\phi}\right)
$$

then

$$
\Omega \cap \mathscr{S}=\tilde{\Omega} \cap \mathscr{S}=\mathscr{S}, \quad \bigcup_{\theta_{1}<\phi<\theta_{1}+\delta}\left\{Q_{\phi}\right\} \subset \partial \Omega \cap \partial \tilde{\Omega} .
$$

Now we will prove $\partial \Omega=\partial \tilde{\Omega}$. Let $P_{0} \in \bigcup_{\theta_{1}<\phi<\theta_{1}+\delta}\left\{Q_{\phi}\right\}$, and let $d=$ $\frac{1}{2} \operatorname{dist}\left(D_{1}, \partial \Omega\right)$. Now we define

$$
s\left(P_{0}\right)=\operatorname{Sup}_{0 \leq r \leq d}\left\{r: B_{r}\left(P_{0}\right) \text { has the property ; for every } P \in B_{r}\left(P_{0}\right) \cap \partial \Omega\right.
$$

there exists a ball $B_{\epsilon}(P)$ such that $B_{\epsilon}(P) \cap \Omega=B_{\epsilon}(P) \cap \tilde{\Omega}$

$$
\text { and } \left.B_{\epsilon}(P) \cap \Omega^{c}=B_{\epsilon}(P) \cap \tilde{\Omega}^{c}\right\} \text {. }
$$

We claim $s\left(P_{0}\right)=d$. Suppose $s<d$. We can pick $\bar{P} \in \partial B_{s}\left(P_{0}\right) \cap \partial \Omega$. Clearly

$$
\bar{P} \in \partial \Omega \cap \partial \tilde{\Omega}, \quad \bar{P} \in \partial(\Omega \cap \tilde{\Omega}) .
$$

Recall $\Omega$ is convex. We can choose a small ball $B(\bar{P})$ such that in local coordinates there exist functions $\psi$ and $\tilde{\psi}$ such that

$$
B(\bar{P}) \cap \Omega=\{(x, y): y<\psi(x)\} \cap B(\bar{P})
$$

and

$$
B(\bar{P}) \cap \tilde{\Omega}=\{(x, y): y<\tilde{\psi}(x)\} \cap B(\bar{P}) .
$$

From the uniqueness of harmonic continuation, it is easy to see that

$$
u_{1}=\tilde{u}_{1} \quad \text { in } B_{s}\left(P_{0}\right) \cap \Omega \cap \tilde{\Omega},
$$

so

$$
u_{1}=\tilde{u}_{1} \quad \text { in } B(\bar{P}) \cap \Omega \cap \tilde{\Omega} .
$$


Let $\bar{P}=\left(x_{0}, \psi\left(x_{0}\right)\right)$. Then there is a small $\epsilon_{1}>0$ such that

$$
(x, \psi(x)),(x, \tilde{\psi}(x)) \in B(\bar{P}) \quad \text { whenever }\left|x-x_{0}\right|<\epsilon_{1} .
$$

We will show $B_{\epsilon_{1}}(\bar{P}) \cap \Omega=B_{\epsilon_{1}}(P) \cap \tilde{\Omega}$. It suffices to show

$$
\psi(x)=\tilde{\psi}(x) \quad \text { for }\left|x-x_{0}\right|<\epsilon_{1} .
$$

If not, there is an $x$ with $\left|x-x_{0}\right|<\epsilon_{1}$ such that $\psi(x)<\tilde{\psi}(x)$ or $\tilde{\psi}(x)<\psi(x)$. Suppose $\psi(x)<\tilde{\psi}(x)$. Then $(x, \psi(x)) \in \tilde{\Omega} \cap B(P)$, and therefore there is a small ball $B_{\epsilon_{2}}(Q)$ where $Q=(x, \psi(x))$ such that $B_{\epsilon_{2}}(Q) \subset B(P) \cap \tilde{\Omega}$. Since

$$
u_{1}=\tilde{u}_{1} \quad \text { in } B_{\epsilon_{2}}(Q) \cap \Omega \subset B(P) \cap \Omega \cap \Omega
$$

and $\tilde{u}_{1}$ is harmonic in $B_{\epsilon_{2}}(Q), u_{1}$ has a harmonic extension to $B_{\epsilon_{2}}(Q)$, and this is not possible because of the bad boundary data $g$. This proves $s\left(P_{0}\right)=d$.

Next we pick $P_{1} \in \partial \Omega \cap \partial B_{d / 2}\left(P_{0}\right)$. Then the same arguments as above show that $s\left(P_{1}\right)=d$, and by repeating this process we conclude $\partial \Omega=\partial \tilde{\Omega}$ or $\Omega=\tilde{\Omega}$. Hence $u_{1}$ has a harmonic extension to

$$
\left(\Omega \backslash \overline{D_{1}}\right) \cup\left(\Omega \backslash \overline{\tilde{D}_{1}}\right) .
$$

We can repeat the same arguments as before for the $k$-rotation of $u_{1}$

$$
\tilde{u}_{1}^{k}(r, \theta)=u_{1}\left(r, \theta-2 k \theta_{0}\right)
$$

for $k=0,1,2, \ldots$ in such a way that any consecutive two rotations $\tilde{u}_{1}^{k}$ and $\tilde{u}_{1}^{k+1}$ play the role of $u_{1}$ and $\tilde{u}_{1}$ respectively, concluding finally that $u_{1}$ has a harmonic extension to

$$
\left(\Omega \backslash \overline{D_{1}}\right) \cup\left(\Omega \backslash \overline{\tilde{D}_{1}}\right) \cup \cdots \cup\left(\Omega \backslash \overline{\tilde{D}_{1}^{k}}\right) .
$$

Here $\tilde{D}_{1}^{k}=\left\{\left(r, \theta+2 k \theta_{0}\right):(r, \theta) \in D_{1}\right\}$.

We claim that this last set is $\Omega \backslash\{0\}$ for some finite $k$. Since $2 \theta_{0}<2 \pi$, there is the positive integer $k_{0}$ such that

$$
\left(2 k_{0}+1\right) \theta_{0}<2 k_{0} \pi \text { and }(2 k+1) \theta_{0} \geq 2 k \pi \text { for } k=0,1, \ldots, k_{0}-1 .
$$

We will show

$$
\boldsymbol{\Omega}=\left(\boldsymbol{\Omega} \backslash \overline{D_{1}}\right) \cup\left(\boldsymbol{\Omega} \backslash \overline{\tilde{D}_{1}}\right) \cup\left(\boldsymbol{\Omega \backslash \overline { \tilde { D } _ { 1 } ^ { k _ { 0 } } }}\right) \cup\{0\} .
$$

In fact, if that is not true, there is an $(r, \theta) \in \Omega$ such that

$$
(r, \theta) \in \bar{D}_{1} \cap \overline{\tilde{D}_{1}} \cap \overline{\tilde{D}_{1}^{k_{0}}} .
$$

From (7) we can get

$$
0 \leq\left(2 k_{0}-1\right) \theta_{0}-2\left(k_{0}-1\right) \pi<\left(2 k_{0}+1\right) \theta_{0}-2\left(k_{0}-1\right) \pi<2 \pi,
$$

where the first inequality comes from the second statement in (7) with $k=k_{0}-1$ and the last inequality comes from the first statement in (7).

Also, we can rewrite the first inequality in (9) as

$$
\theta_{0} \leq 2 k_{0} \theta_{0}-2\left(k_{0}-1\right) \pi
$$


If $(r, \theta) \in \overline{\tilde{D}_{1}^{k_{0}}}$, then

$$
2 k_{0} \theta_{0}-2\left(k_{0}-1\right) \pi \leq \theta \leq\left(2 k_{0}+1\right) \theta_{0}-2\left(k_{0}-1\right) \pi .
$$

This implies, using (9), that $\theta \geq \theta_{0}$. But $(r, \theta) \in \overline{D_{1}}$ implies $\theta \leq \theta_{0}$. Hence $\theta=\theta_{0}$. But then $\left(r, \theta_{0}\right)$ cannot belong to ${\overline{D_{1}}}_{1}$, a contradiction. This proves identity (8).

Hence we have extended $\left.u_{1}\right|_{\Omega \backslash D_{1}}$ harmonically into $\Omega \backslash\{0\}$. Since we already know $\left.u_{1}\right|_{\Omega \backslash D_{1}}$ has a harmonic extension to $B_{\epsilon}(0)$, we conclude that $\left.u_{1}\right|_{\Omega \backslash D_{1}}$ has a harmonic extension to $\Omega$. Let $v$ denote this harmonic extension to $\Omega$. Because of the uniqueness in the Dirichlet problem, $v=u_{1}$ in $D_{1}$. Hence the transmission conditions (3) imply $\frac{\partial u_{1}}{\partial n}=0$ on $\partial D_{1}$, so $u_{1}$ is a constant in $\Omega$. Hence $g \equiv 0$, a contradiction.

Lemma 3 below and the ideas of Theorem 1 give us the following result in $R^{n}$.

Theorem 2. Let $n \geq 2$. Assume $D_{1}$ and $D_{2}$ are convex polyhedra. Under the assumption on Theorem $1, D_{1}=D_{2}$.

Here we include the proof of Lemma 3, the ideas of which were already in Friedman and Isakov [1].

Lemma 3. Let $n \geq 2$. Assume $u$ is a solution to (1). Let $\gamma$ be an edge of $D$ with angle $\theta_{1}$. Suppose that there is $a Q \in \gamma$ with $B_{r_{0}}(Q) \subset \Omega$ for some small $r_{0}>0$ such that $\left.u\right|_{B_{r_{0}}(Q) \backslash D}$ has harmonic continuation to the whole $B_{r_{0}}(Q)$. Then there is a rotation $R_{2 \theta_{1}}$ on the 2-dimensional plane $E$ perpendicular to $\gamma$ at $Q$ such that $u(x)=u\left(R_{2 \theta_{1}}(x)\right)$ for all $x \in B_{\epsilon}$ and some $\epsilon<r_{0}$.

Proof. We will give the proof for $n \geq 3$, with the obvious changes for $n=2$. We can assume $Q$ is the origin of $R^{n}, \gamma$ is the $(n-2)$-dimensional segment

$$
\gamma=\left\{\left(x_{1}, x_{2}, \ldots, x_{n}\right) \in R^{n}: x_{1}=x_{2}=0\right\} \cap B_{1}(0)
$$

obtained as the intersection $F_{1} \cap F_{2}$ of two faces of the polygon, $E$ is the twodimensional plane $x_{3}=\cdots=x_{n}=0$ in which we introduce polar coordinates $(r, \theta)$ and

$$
E \cap D \cap B_{r_{0}}(0)=\left\{(r, \theta): 0<r<r_{0}, 0<\theta<\theta_{1}\right\} .
$$

We will first observe that if $\left.u\right|_{B_{r_{0}} \backslash D}$ has a harmonic continuation to $B_{r_{0}}(0)$, then there is a small $\epsilon>0$ with $\epsilon<r_{0}$ such that $\left.u\right|_{B_{\epsilon} \cap D}$ has a harmonic continuation into the whole $B_{\epsilon}(0)$. To see this, let $u^{\text {ext }}$ denote the harmonic extension of $\left.u\right|_{B_{r_{0}} \backslash D}$ to $B_{r_{0}}$, and consider the Cauchy problem

$$
\left\{\begin{array}{l}
\Delta w=0 \text { in } B_{\epsilon}(0), \\
w=u, \frac{\partial w}{\partial n}=\frac{1}{2} \frac{\partial u^{\mathrm{ext}}}{\partial n} \quad \text { on } \pi_{1} \cap B_{\epsilon}(0),
\end{array}\right.
$$

where $\pi_{1}$ is the $(n-1)$-dimensional plane containing the face $F_{1}$. Since $u^{\mathrm{ext}}$ is analytic on $B_{\epsilon}(0)$, by the Cauchy-Kovalevski Theorem there exists a unique analytic solution to (12) on $B_{\epsilon}(0)$ if $\epsilon$ is small enough. But $\left.u\right|_{B_{\epsilon} \cap D}$ satisfies the same equation in $D \cap B_{\epsilon}(0)$ with the same Cauchy data on $F_{1} \cap B_{\epsilon}(0)$. Therefore, $w=u$ in $B_{\epsilon} \cap D$ by the uniqueness of harmonic extension, and we will denote $u^{\text {int }}=w$ in $B_{\epsilon}$. 


\section{Consider now}

$$
v_{1}=u^{\mathrm{ext}}-u^{\mathrm{int}}, \quad v_{2}=u^{\mathrm{ext}}-2 u^{\mathrm{int}} .
$$

Because of the continuity of the solution across the interfaces and the jump relations $(3)$ on $\partial D$, we have

$$
v_{1}=0 \text { and } \frac{\partial v_{2}}{\partial \theta}=0 \quad \text { for } 0<r<\tilde{\epsilon}, \theta=0, \theta_{1} .
$$

Since $v_{1}, v_{2}$ are harmonic in $B_{\tilde{\epsilon}}(0)$, we can write for every $x^{\prime}=\left(x_{3}, \ldots, x_{n}\right) \in$ $R^{n-2}$

$$
\begin{aligned}
& v_{1}\left(r, \theta, x^{\prime}\right)=\sum_{k=0}^{\infty}\left(a_{k}^{1}\left(x^{\prime}\right) \cos (k \theta)+b_{k}^{1}\left(x^{\prime}\right) \sin (k \theta)\right) r^{k}, \\
& v_{2}\left(r, \theta, x^{\prime}\right)=\sum_{k=0}^{\infty}\left(a_{k}^{2}\left(x^{\prime}\right) \cos (k \theta)+b_{k}^{2}\left(x^{\prime}\right) \sin (k \theta)\right) r^{k} .
\end{aligned}
$$

From (13), we obtain

$$
\begin{aligned}
& a_{k}^{1}\left(x^{\prime}\right)=0, \quad b_{k}^{1} \sin \left(k \theta_{1}\right)=0 \quad \text { for } k=1,2, \ldots, \\
& a_{k}^{2}\left(x^{\prime}\right) \sin \left(k \theta_{1}\right)=0, \quad b_{k}^{2}\left(x^{\prime}\right)=0 \quad \text { for } k=1,2, \ldots .
\end{aligned}
$$

Hence $v_{i}\left(r, \theta+2 \theta_{1}, x^{\prime}\right)=v_{i}\left(r, \theta, x^{\prime}\right)$ for $i=1,2$. Therefore, the same hold for $u^{\text {ext }}$ and $u^{\text {int }}$, and the conclusion of Lemma 3 follows.

Remark. When $D_{1}, D_{2}$ in Theorem 1 are general polyhedra, the above proof shows convex hull $\left(D_{1}\right)=$ convex hull $\left(D_{2}\right)$.

\section{REFERENCES}

1. A. Friedman and V. Isakov, On the uniqueness in the inverse conductivity problem with one measurement, Indiana Univ. Math. J. 38 (1989).

2. D. Gilbart and N. Trudinger, Elliptic partial differential quations of second order, 2nd ed., Springer-Verlag, New York, 1983.

3. O. A. Ladyzenskaja and N. N. Ural'zeva, Linear and quasi-linear elliptic equations, Academic Press, London, 1968. SPAIN

Departamento de Matemáticas, Universidad Autónoma de Madrid, 28049 Madrid,

School of Mathematics, University of Minnesota, Minneapolis, Minnesota 55455

G.A.R.C. Seoul National University, Seoul, Korea

Current address: Department of Mathematics, Pohang Institute of Science and Technology, Pohang, 790-600 Korea 